\title{
Identification of differentially expressed miRNAs and mRNAs in synovial of osteoarthritis via RNA-sequencing
}

\author{
Yu Zhou ${ }^{\dagger}$, Zhicong Wang ${ }^{\dagger}, X_{i}$ Chen ${ }^{*}$, Jianjun Zhang, Ling Yang, Shuping Liu and Yuehong Liu* (D)
}

\begin{abstract}
Background: Osteoarthritis $(\mathrm{OA})$ is the most common form of arthritis and a leading cause of disability. This study attempted to investigate the key mRNAs and miRNAs related to OA.

Patients and methods: From April 17th, 2018 to May 17th, 2018, five patients with OA and three normal controls were enrolled in this present study. To identify the differentially expressed mRNAs (DEmRNAs) and miRNAs (DEmiRNAs) between patients with OA and normal controls, RNA-sequencing was performed. Then, DEmiRNAtarget DEmRNAs analysis and functional annotation of DEmiRNA-target DEmRNAs were performed. To validate the RNA-sequencing results, quantitative real time-PCR (RT-PCR) and western blot analysis were performed as well.

Results: A total of 1068 DEmRNAs, 21 DEmiRNAs and 395 DEmiRNA-DEmRNA pairs were identified in synovial tissues of patients with OA. The functional annotation of DEmiRNA-target DEmRNAs revealed that Pathways in cancer and PI3K-Akt signaling pathway were significantly enriched Kyoto Encyclopedia of Genes and Genomes (KEGG) pathways. QRT-PCR and western blot results revealed that except for TLR7, the expression level of the others was consistent with the RNA-sequencing results, generally.
\end{abstract}

Conclusion: The findings of this present study may provide new clues for the roles of DEmRNAs and DEmiRNAs in the pathogenesis of OA.

Keywords: Osteoarthritis (OA), mRNA, miRNA, RNA-sequencing, Functional annotation

\section{Introduction}

Osteoarthritis (OA) is the most frequent musculoskeletal disease and leads to functional decline and loss in quality of life [1]. Currently, there is no effective treatment to prevent the initiation and progression of the disease while the severity of OA often worsens with age [2]. Reportedly, it estimates that among population over 60 years old, there was $9.6 \%$ of men and $18 \%$ of women suffer from symptomatic OA in the worldwide [3]. Although the disease is with a trait of destruction of articular cartilage, pathological changes in subchondral bone and associated synovitis, the pathogenesis is poor understanding which is thought to be multifactorial [4]. Inability to diagnose

\footnotetext{
*Correspondence: cxchenxi007@163.com; liuyuehong_doctor@163.com ${ }^{\dagger}$ Yu Zhou and Zhicong Wang contributed equally to this work.

Department of Orthopedics, People's Hospital of Deyang City, No. 173, Taishan North Road, Jingyang District, Deyang 618000, Sichuan, China
}

early and poorly understanding of the pathophysiology makes early diagnosis to be a key factor in the prevention and management of disease [5]. Recently, molecular biology has been reported to play a vital role in explaining its disease pathophysiology, and gene regulation has been demonstrated to be involved in driving an imbalance between the expression of catabolic and anabolic factors, leading eventually to osteoarthritic cartilage degeneration [6]. There is increasing attention on the influence of dysregulation at a molecular level on the pathogenic process.

MicroRNAs (miRNAs) have been detected widely in eukaryotes to play roles in regulating growth, development, differentiation, and metabolism in model organisms which are short (approximately 22 nucleotides), non-coding, RNA regulators of gene expression [7]. Little was known of the function of miRNAs at the stage when it is first identified in the early 1990's by Lee 
et al. (in Caenorhabditis elegans) [8]. Due to the link between alterations in miRNA expression levels and multifarious disease processes have been linked, aberrant expression in pathological states has drawn public attention [9]. Studies based on highly specific patterns of miRNA expression correlate with development and several diseases have revealed the potential for therapeutic manipulation of miRNAs [10, 11]. Recently, a large number of studies have been done to explore miRNAs and genes associated with OA [5, 6, 12-14]. Even so, studies on biomarkers for OA are still urgent to be performed.

In this study, differentially expressed miRNAs (DEmiRNA) and mRNAs (DEmRNAs) in synovial tissues of patients with OA were identified by RNAsequencing. DEmiRNA-target DEmRNAs analysis and functional annotation of DEmiRNA-target DEmRNAs were performed. Quantitative real time-PCR (RTPCR) and western blot analysis were performed to validate the RNA-sequencing results. In doing so, we hope this study could represent a new avenue to understand the pathogenesis and develop potential biomarkers for OA.

\section{Materials and methods}

\section{Patients and samples}

Five patients with $\mathrm{OA}$ and three normal controls were recruited in this study from April 17th, 2018 to May 17th, 2018. According to the criteria of the American College of Rheumatology, OA were diagnosed [15]. Healthy employees with no symptoms or signs of OA, or any other type of arthritis, or any painful condition of the joints, were included as controls. Beside, participants with no personal or family history of OA were selected as control subjects. The participants with history of joint diseases, including inflammatory arthritis (rheumatoid arthritis or any other autoimmune disease), post-traumatic or post-septic arthritis, poliomyelitis, skeletal dysplasia, were excluded. Radiographic evaluation of all participants were performed. Table 1 displayed the detailed information of all these participants. The written informed consent for use of their samples from every participant were provided in the present study. Ethical approval for this study was granted by the ethics committee of People's Hospital of Deyang City (2017-043). Synovial tissues of every participant were obtained.

\section{RNA isolation and sequencing}

Following the manufacturer's protocol, we used TRIzol reagent (Invitrogen, Carlsbad, CA, USA) to isolate total RNA from samples. The concentration and purity of RNA was determined with Nanodrop ND-2000 spectrophotometer (Thermo Fisher Scientific, Wilmington, DE, USA), and the integrity of RNA was confirmed via a $2 \%$ agarose gel. With an Agilent 2100 Bioanalyzer (Agilent, Palo Alto, CA, USA), the RNA integrity number (RIN) value was obtained. With QiaQuick PCR Purification Kit, the mRNA library was constructed. The 18-30 nt RNA was obtained from the total RNA. By using TruseqTM Small RNA Sample Prep Kit, adapter ligation and reverse transcription polymerase chain reaction (PCR) were performed to obtain the cDNA. Sequencing was performed based on HiSeq $\mathrm{x}$-ten platform (Illumina) and SE50, BGIseq, respectively.

\section{Identification of DEmRNAs in patients with OA compared with normal controls}

The clean reads were aligned with the human reference genome, Ensemble GRCh38.p7 (ftp://ftp.ncbi.nlm.nih. gov/genomes/Homo_sapiens) by using TopHat release 2.2.1 (http://tophat.cbcb.umd.edu/). With Cuffquant version 2.2.1 (http://cufflinks.cbcb.umd.edu/), expression of mRNAs was normalized and outputted. To determine the transcription abundance of mRNAs, fragments per Kilobase of exon per million fragments mapped (FPKM) was used. With Cuffdiff version 2.2.1 (http://cufflinks. cbcb.umd.edu/), FPKMs of mRNAs were calculated. Differentially expressed mRNAs (DEmRNAs) were identified with $p<0.05$ and $\left|\log _{2} \mathrm{FC}\right|>1$. Hierarchical clustering analysis of DEmRNAs was conducted with $\mathrm{R}$

Table 1 Patient characteristics

\begin{tabular}{|c|c|c|c|c|c|c|c|c|}
\hline Index & Weight (kg) & Height (cm) & Kellgren Lawrence grade (n) & Disease duration & Blood glucose (mmol/L) & CRP (mg/L) & $\mathrm{ESR}(\mathrm{mm} / \mathrm{h})$ & Part \\
\hline Case 1 & $56-75$ & $136-165$ & 4 & 8 years & 5.63 & 3.44 & 48 & Left knee \\
\hline Case 2 & & & 1 & 2 months & 5.98 & 2.9 & 8 & Left knee \\
\hline Case 3 & & & 4 & 30 years & 5.08 & & 14 & Right knee \\
\hline Case 4 & & & 2 & 10 years & 6.2 & 27.94 & 16 & Left knee \\
\hline Case 5 & & & 4 & 4 years & 5.1 & 0 & 16 & Right knee \\
\hline Control 1 & $46-60$ & $155-165$ & - & $3 \mathrm{~h}$ & & - & - & Right knee \\
\hline Control 2 & & & - & $2 \mathrm{~h}$ & 4.62 & - & - & Right knee \\
\hline Control 3 & & & - & 1 day & 5.2 & - & - & Left knee \\
\hline
\end{tabular}

CRP C-reactive protein, ESR Erythrocyte sedimentaition ratio 
package "pheatmap". The flow chart of the analyses was showed in Figure S1.

\section{Functional annotation of DEmRNAs between patients with $\mathrm{OA}$ and normal controls}

To further research the biological function of DEmRNAs, Gene Ontology (GO) function and Kyoto Encyclopedia of Genes and Genomes (KEGG) pathway enrichment analyses of the DEmRNAs between patients with OA and normal controls were performed by DAVID 6.8 (https://david.ncifcrf.gov/). A value of $p<0.05$ was defined as the criteria of statistical significance.

\section{Identification of DEmiRNAs in patients with OA compared with normal controls}

With Bowtie (bowtie-bio.sourceforge.net), the alignment between the cleaned miRNA sequencing reads was aligned to the human genome (GRCh38.p7 assembly) based on Genome human UCSC reference annotation. With miRDeep2 (https://www.mdc-berlin.de/8551903/ en/), the transcription abundance of miRNAs was determined. Based on the read count of each sample, the DEmiRNAs in OA compared to normal controls were calculated with an R-bioconductor package, DESeq2 (http://www.bioconductor.org/packages/release/bioc/ html/DESeq2.html). For DEmiRNAs in OA compared to normal controls, the threshold was defined as base Mean $>100, p<0.05$ and $\left|\log _{2} \mathrm{FC}\right|>2$. With $\mathrm{R}$ package "pheatmap", hierarchical clustering analysis of DEmRNAs was conducted.

\section{DEmiRNA-target DEmRNAs analysis}

Given miRNAs tend to decrease the expression of their target mRNAs, we selected target genes from DEmRNAs that expressed inversely with that of miRNA for further research. Firstly, the putative targeted DEmRNAs of DEmiRNAs were predicted by six bioinformatic algorithms (RNA22, miRanda, miRDB, miRWalk, PICTAR2 and Targetscan). Then, with miRWalk, the confirmed targeted DEmRNAs of DEmiRNAs were obtained. Thirdly, the confirmed DEmiRNA-DEmRNA pairs were derived from miRWalk and the DEmiRNA-DEmRNA pairs recorded by $\geq 4$ algorithms. Based on the obtained DEmiRNA-DEmRNA pairs, DEmiRNA-DEmRNA interaction networks between $\mathrm{OA}$ and normal controls were constructed by using Cytoscape software (http://www. cytoscape.org/).

\section{Functional annotation of DEmiRNA targets}

To further research the biological function of the target DEmRNAs of DEmiRNAs, GO analysis was performed $t$ by using Gorilla (http://cbl-gorilla.cs.technion.ac.il/) with a $p$-value $<0.01$, and KEGG pathway analysis was performed by using webgestalt (http://www.webgestalt. org/) with a $p$-value $<0.05$.

\section{Quantitative real time-PCR (RT-PCR) and western blot analysis}

Eight synovial tissues were obtained from four patients with $\mathrm{OA}$ and four normal controls. The written informed consent for use of their samples from every participant were provided in the present study. Ethical approval for this study was granted by the ethics committee of People's Hospital of Deyang City (2017-043).

Total RNA was isolated with the Trizol reagent (Invitrogen, USA). The qRT-PCR reactions were performed based on SuperReal PreMix Plus (Invitrogen, USA) in ABI 7300 Real-time PCR Detection System. With $2^{-\Delta \Delta C t}$ method, relative gene expression was determined. The human GAPDH and ACTB were used as endogenous controls for mRNA expression, and the human hsa-U6 was used as endogenous controls for miRNA expression in analysis.

Synovial tissues were lysed on ice with RIPA lysis buffer, and then the supernatants were collected by centrifugation at $12,000 \mathrm{rpm}$ at $4{ }^{\circ} \mathrm{C}$ for $30 \mathrm{~min}$. The protein concentrations were detected with $\mathrm{BCA}$ protein assay. The protein extracts were separated by $10 \%$ SDS-PAGE, transferred onto PVDF membranes, and probed using primary and then secondary antibodies. The primary antibodies were as follows: rabbit anti-GAPDH, TIMP3 Antibody, CTSS Antibody and TLR7 Antibody. The blots were visualized with ECL reagent.

\section{Results}

DEmRNAs and DEmiRNAs between patients with OA and normal controls

Expression profiling of mRNA and miRNA extracted from five patients with $\mathrm{OA}$ and three normal controls identified 1068 DEmRNAs (516 up-regulated and 552 down-regulated DEmRNAs) and 21 DEmiRNAs (6 upregulated and 15 down-regulated DEmiRNAs). The top 10 up- and down-regulated DEmRNAs and all DEmiRNAs were showed in Table 2 and Table 3, respectively. Hierarchical clustering analysis of top 50 up- and downregulated DEmRNAs and DEmiRNAs was displayed in Fig. 1 and Fig. 2, respectively. The raw-data have been uploaded to Gene Expression Omnibus (GEO) (GSE143514, https://www.ncbi.nlm.nih.gov/geo/query/ acc.cgi?acc $=$ GSE143514).

\section{Functional annotation of DEmRNAs between patients with $\mathrm{OA}$ and normal controls}

Protein phosphorylation ( $p=1.84 \mathrm{E}-05)$, defense response to virus $(p=3.54 \mathrm{E}-05)$, positive regulation of transcription from RNA polymerase II promoter $(p=4.08 \mathrm{E}-05)$, extracellular space $(p=7.09 \mathrm{E}-24)$ and heparin binding 
Table 2 Top 10 up- and down-regulated DEmRNAs between patients with $\mathrm{OA}$ and normal controls

\begin{tabular}{|c|c|c|c|c|}
\hline ID & Symbol & $\log _{2} \mathrm{FC}$ & $p$-value & Regulation \\
\hline 91,937 & TIMD4 & 3.81464 & $5.00 \mathrm{E}-05$ & up \\
\hline 57,152 & SLURP1 & 3.86063 & 0.0037 & up \\
\hline 27,344 & PCSK1N & 3.93602 & 0.00065 & up \\
\hline 55,600 & ITLN1 & 4.00229 & 0.0016 & up \\
\hline 25,791 & NGEF & 4.17476 & $5.00 \mathrm{E}-05$ & up \\
\hline 140,578 & CHODL & 4.2872 & 0.01285 & up \\
\hline $100,423,062$ & IGLL5 & 4.79462 & 0.0298 & up \\
\hline 1446 & CSN1S1 & 5.81655 & $5.00 \mathrm{E}-05$ & up \\
\hline 91,937 & TIMD4 & 3.81464 & $5.00 \mathrm{E}-05$ & up \\
\hline 57,152 & SLURP1 & 3.86063 & 0.0037 & up \\
\hline 4316 & MMP7 & -7.00868 & 0.00845 & down \\
\hline 8862 & APLN & -5.19773 & $5.00 \mathrm{E}-05$ & down \\
\hline 3381 & IBSP & -4.29821 & 0.00195 & down \\
\hline 54,360 & CYTL1 & -4.23704 & $5.00 \mathrm{E}-05$ & down \\
\hline 6781 & STC1 & -4.21422 & $5.00 \mathrm{E}-05$ & down \\
\hline 3569 & IL6 & -4.15517 & $5.00 \mathrm{E}-05$ & down \\
\hline 4321 & MMP12 & -4.03152 & 0.0094 & down \\
\hline 90,249 & UNC5A & -3.84362 & 0.03125 & down \\
\hline 7980 & TFPI2 & -3.81017 & 0.0099 & down \\
\hline 7941 & PLA2G7 & -3.78853 & 0.02245 & down \\
\hline
\end{tabular}

DEmRNAs Differentially expressed mRNAs, FC Fold change $(p=2.03 \mathrm{E}-12)$ were significantly enriched $\mathrm{GO}$ terms in OA (Fig. 3a-c). Staphylococcus aureus infection ( $p=$ 5.90E-12), Leishmaniasis $(p=4.66 \mathrm{E}-11)$ and HTLV-I infection $(p=1.84 \mathrm{E}-08)$ were significantly enriched KEGG pathways in OA (Fig. 3d).

\section{DEmiRNA-target interactions}

A total of 395 DEmiRNA-DEmRNA pairs, including 376 DEmiRNA-DEmRNA pairs which were predicted by $\geq 4$ algorithms and 48 validated DEmiRNA-DEmRNA pairs derived from the miRWalk, were obtained (Fig. 4). Among which, hsa-miR-17-5p (degree $=62)$, hsa-miR20b-5p (degree $=56)$ and hsa-miR-106a-5p $($ degree $=52)$ were the top three DEmiRNAs that covered most DEmRNAs.

\section{Functional annotation of DEmiRNA targets}

Base on GO enrichment analysis, RNA processing ( $p=$ $5.80 \mathrm{E}-04)$, response to activity $(p=9.04 \mathrm{E}-04)$, nucleus $(p=3.56 \mathrm{E}-04)$ and alpha-tubulin binding ( $p=5.80 \mathrm{E}-04)$ were significantly enriched GO terms in OA (Fig. 5a-c). According to the KEGG pathway enrichment analysis, the DEmiRNA-target DEmRNAs were significantly enriched in Pathways in cancer $(p=3.36 \mathrm{E}-02)$ and PI3KAkt signaling pathway ( $p=4.67 \mathrm{E}-02)$ (Fig. 5d-e) [16].

Table 3 DEmiRNAs between patients with OA and normal controls

\begin{tabular}{|c|c|c|c|c|}
\hline miRNA|precursor & baseMean & $\log _{2} \mathrm{FC}$ & $p$-value & Regulation \\
\hline hsa-miR-106a-5p|hsa-mir-106a & 492.1284 & -3.19961 & 0.000226 & Down \\
\hline hsa-miR-1246|hsa-mir-1246 & 258.1751 & -2.0786 & 0.011492 & Down \\
\hline hsa-miR-144-3p|hsa-mir-144 & $17,728.54$ & -3.31783 & 0.049975 & Down \\
\hline hsa-miR-17-5p|hsa-mir-17 & 7597.817 & -2.22172 & 0.004888 & Down \\
\hline hsa-miR-18b-5p|hsa-mir-18b & 109.0147 & -2.1827 & 0.012516 & Down \\
\hline hsa-miR-20b-5p|hsa-mir-20b & 1693.174 & -3.26326 & 0.000196 & Down \\
\hline hsa-miR-363-3p|hsa-mir-363 & 4384.713 & -2.63676 & 0.000153 & Down \\
\hline hsa-miR-451a|hsa-mir-451a & $5,294,654$ & -2.72244 & 0.005101 & Down \\
\hline hsa-miR-548ad-5p|hsa-mir-548ad & 110.1592 & -2.59423 & 0.000577 & Down \\
\hline hsa-miR-548ad-5p|hsa-mir-548ay & 161.6666 & -2.04144 & 0.001393 & Down \\
\hline hsa-miR-548ae-5p|hsa-mir-548ad & 110.1592 & -2.59423 & 0.000577 & Down \\
\hline hsa-miR-548ae-5p|hsa-mir-548ay & 161.6666 & -2.04144 & 0.001393 & Down \\
\hline hsa-miR-548ay-5p|hsa-mir-548ay & 161.6666 & -2.04144 & 0.001393 & Down \\
\hline hsa-miR-675-3p|hsa-mir-675 & 115.7026 & -2.11926 & 0.001639 & Down \\
\hline hsa-miR-96-5p|hsa-mir-96 & 2277.136 & -3.01869 & 0.000203 & Down \\
\hline hsa-miR-122-3p|hsa-mir-122 & 182.4923 & 8.118732 & 0.000981 & Up \\
\hline hsa-miR-122-5p|hsa-mir-122 & 2707.5 & 6.661302 & 2.67E-05 & Up \\
\hline hsa-miR-137|hsa-mir-137 & 351.0572 & 2.317024 & 0.002847 & Up \\
\hline hsa-miR-138-5p|hsa-mir-138-1 & 1356.231 & 2.898852 & 0.000224 & Up \\
\hline hsa-miR-138-5p|hsa-mir-138-2 & 307.7424 & 2.781289 & 0.000927 & Up \\
\hline hsa-miR-215-5p|hsa-mir-215 & 518.2554 & 5.316112 & 0.001199 & Up \\
\hline
\end{tabular}




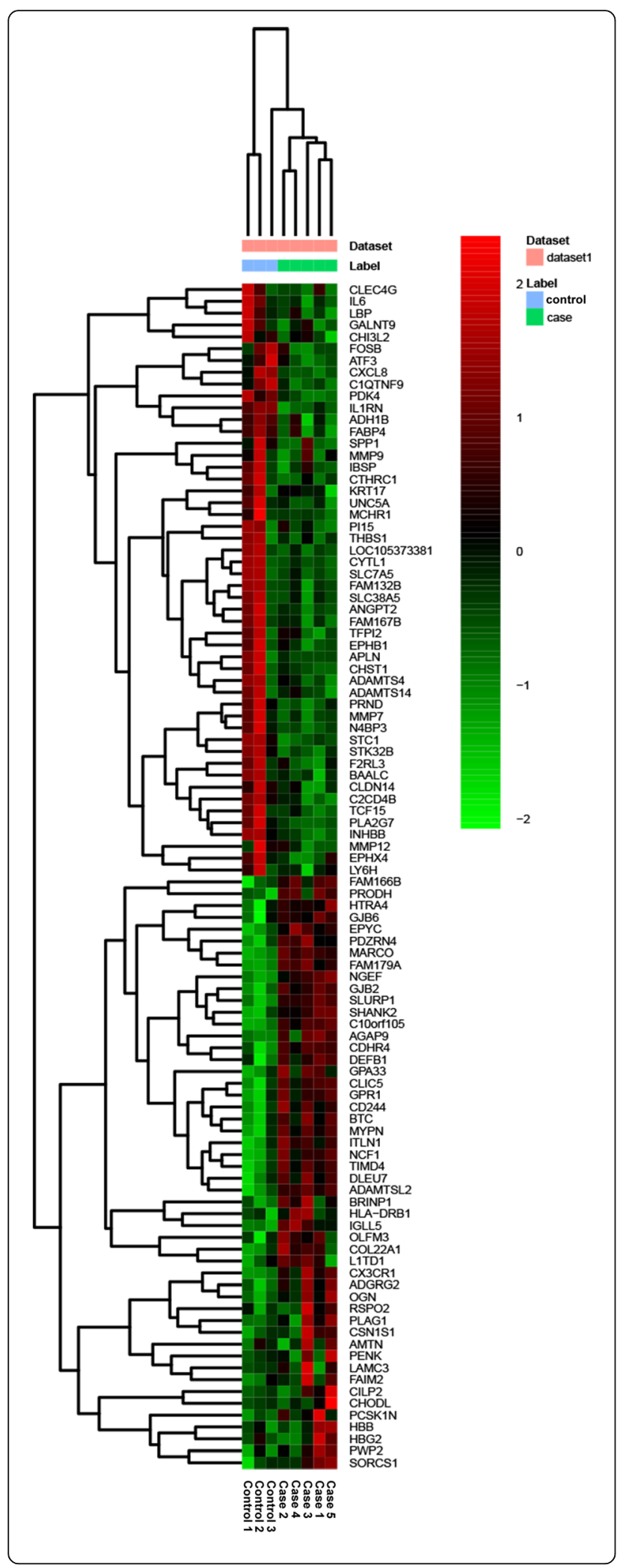

Fig. 1 The heatmap of top 50 up- and down-regulated DEmRNAs between OA and normal controls. Row and column represented DEmRNAs and tissue samples, respectively. The color scale represented the expression levels. The red and green color represented the up- and down-regulated

The results of qRT-PCR and western blot

Three DEmRNAs (including TLR7, CTSS and TIMP3) and two DEmiRNAs (including hsa-miR-17-5p and hsa-miR-20b-5p) were selected to use for qRT-PCR validation. Based on the results of RNA sequencing, TLR7, CTSS and TIMP3 were up-regulated while hsa-miR-17-5p and hsa-miR-20b-5p were downregulated in OA. Except for TLR7, expression of the others in the qRT-PCR results was consistent with that in this present study, generally (Fig. 6a). Western blot results revealed that the protein level of CTSS and TIMP3 were up-regulated while the protein level of TLR7 was down-regulated in OA (Fig. 6b).

\section{Discussion}

OA is a progressive disease with a long silent period, which shows signs of cartilage degradation, mild-tomoderate synovial inflammation and altered bone structure, resulting in severe destruction and impaired function of the affected joints [17]. This study performed RNA-sequencing to attempt to obtain the key miRNAs and mRNAs associated with OA. Here, we discussed two DEmiRNAs (including hsa-miR-17$5 p$ and hsa-miR-20b-5p), which were the top two DEmiRNAs that covered most DEmRNAs, and three DEmRNAs (including TLR7, CTSS and TIMP3), which were target genes of the two DEmiRNAs mentioned above.

Toll like receptor 7 (TLR7), a member of the Tolllike receptor (TLR) family, encoded by TLR7, plays a fundamental role in pathogen recognition and activation of innate immunity [18]. From Drosophila to humans, TLRs are highly conserved and share structural and functional similarities [18]. In humans, the TLR family, a set of 10 type I transmembrane receptors, have specificity for different types of pathogenassociated molecular patterns (PAMPs) [19]. PAMPs are expressed on infectious agents, and mediate the production of cytokines necessary for the development of effective immunity [19]. In humans, ten TLRs have been identified, and TLR-1, TLR-2, TLR-4, TLR-5, TLR-6, and TLR-10 are expressed on the cell surface while TLR-3, TLR-7, TLR-8, and TLR-9 are expressed in endosomes [20]. TLR-7 recognizes single-stranded RNA (ssRNA) in endosomes and is activated by the synthetic antiviral compound imiquimod [21]. TLR activation has been demonstrated to be involved in the 


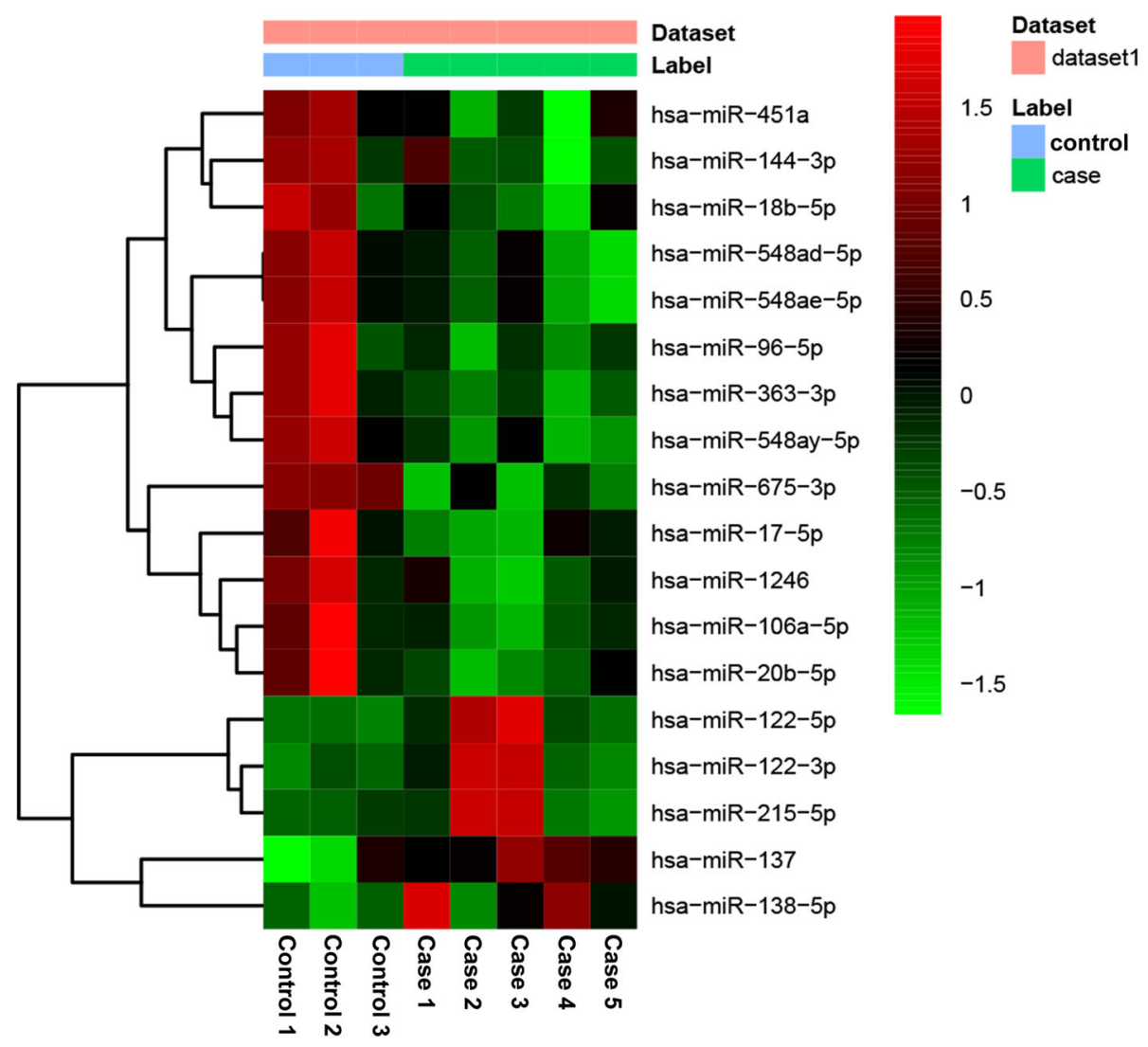

Fig. 2 The heatmap of DEmiRNAs between OA and normal controls. Row and column represented DEmiRNAs and tissue samples, respectively. The color scale represented the expression levels. The red and green color represented the up- and down-regulated

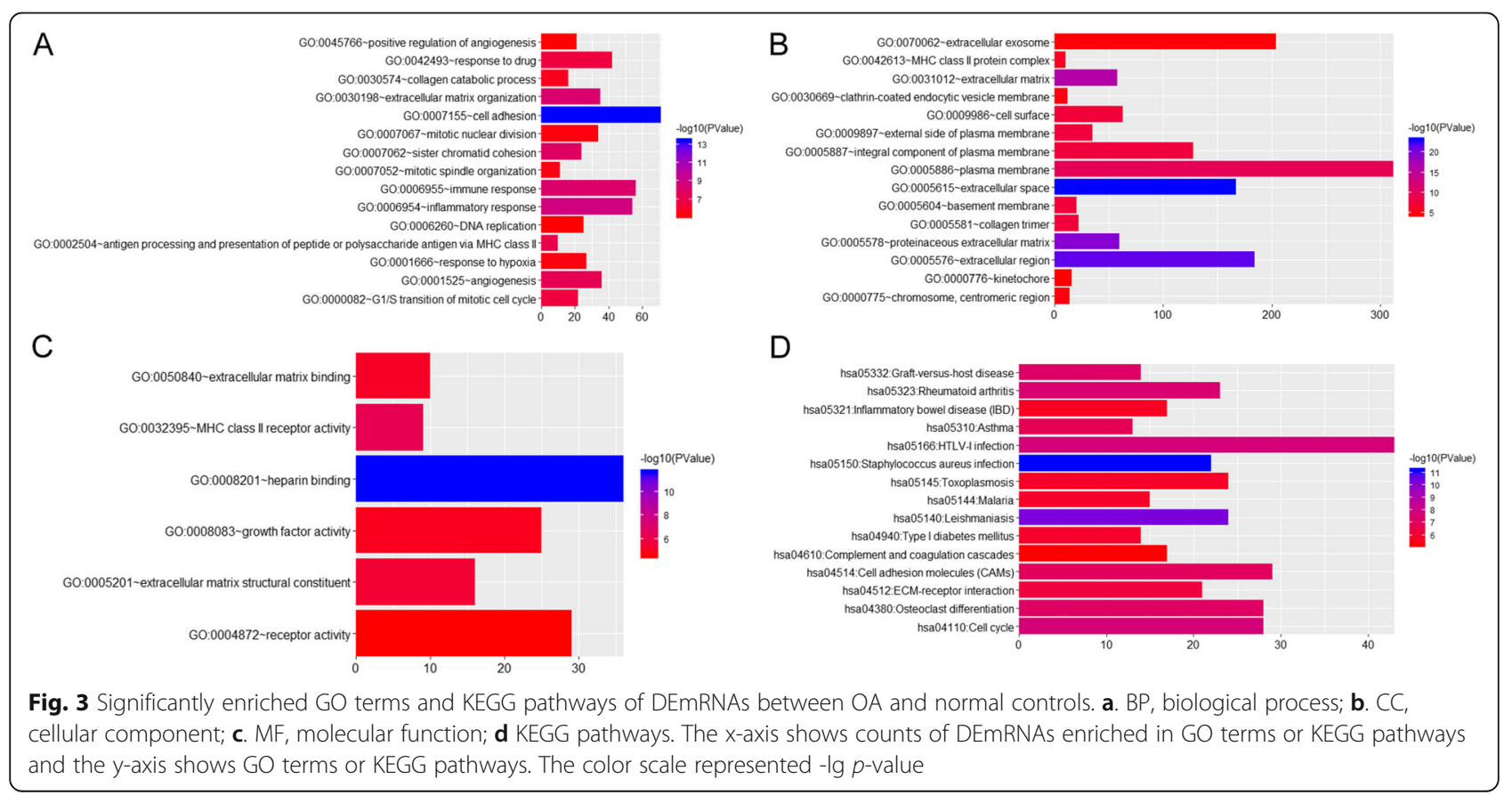




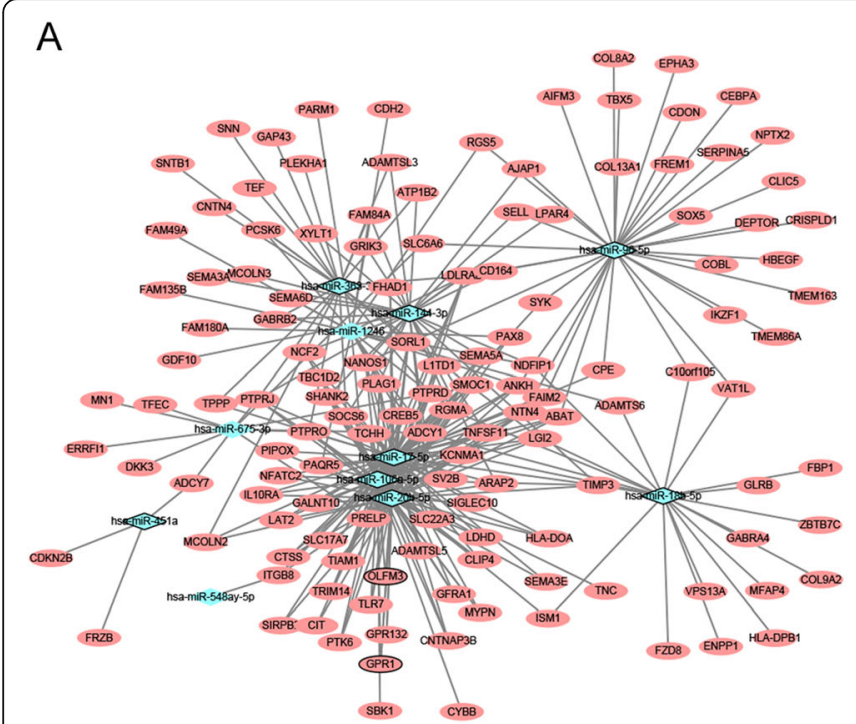

B

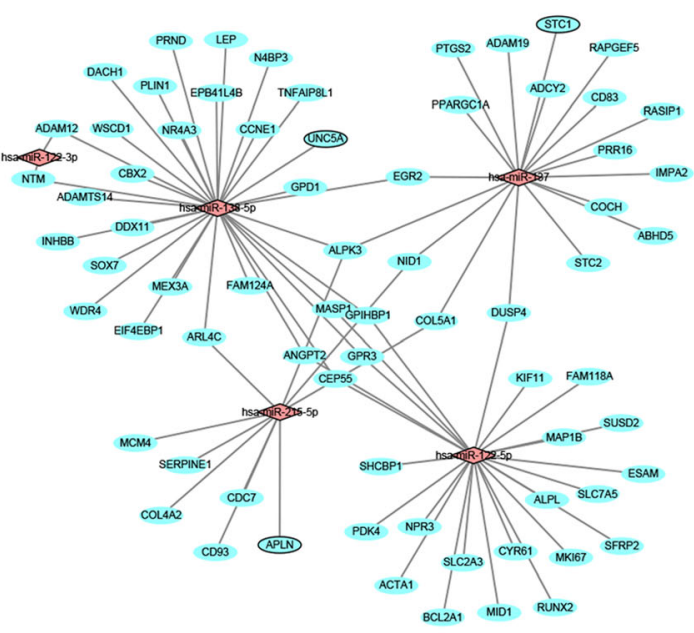

Fig. 4 DEmiRNA-DEmRNA interaction network. a Interaction network between down-regulated DEmiRNAs and up-regulated DEmRNAs; b Interaction network between up-regulated DEmiRNAs and down-regulated DEmRNAs. The rhombic nodes and elliptical nodes indicate DEmiRNAs and DEmRNAs, respectively. Red and green color represent up-regulation and down-regulation, respectively.

development of synovitis, degeneration of cartilage, and susceptibility to disease in OA [22]. Chamberlain et al. suggested that the expression of TLR7 may be a predictor for rheumatoid arthritis (RA) disease activity and anti-TNF $\alpha$ responsiveness, and targeting TLR7 may suppress chronic progression of RA [23]. Mar et al. indicated that the expression level of TLR7 was higher in OA fibroblast-like synoviocytes [24]. Due to TLR7 was found to be up-regulated in OA in our study, we speculated that TLR7 may involve in OA. However, down-regulated TLR7 was found in the results of qRT-PCR and western blot, its exact role in $\mathrm{OA}$ need to be confirmed in the further study.

CTSS (cathepsin S), encoded by CTSS, is a member of the peptidase $\mathrm{C} 1$ family [25]. In addition, CTSS is a lysosomal cysteine proteinase that may participate in the degradation of antigenic proteins to peptides for presentation on MHC class II molecules [25]. Appleton et al. identified increased expression of CTSS in the OA model [26]. The study of Lambert et al. showed that CTSS was significantly up-regulated in inflamed synovial biopsy samples which was confirmed at the protein level by using immunohistochemistry, and it was also significantly up-regulated in cartilage catabolism pathway based on their results [27]. In agreement with previous studies, we observed that CTSS was up-regulated in patients with OA which may imply the importance of CTSS in OA.
TIMP3 (full name, TIMP metallopeptidase inhibitor 3 ), is a member of TIMP family which is inhibitors of the matrix metalloproteinases, a group of peptidases implicated in degradation of the extracellular matrix (ECM) [28]. In bone, TIMP-3 plays the role as a local cytokine, regulating bone metabolism through suppressing osteoblast differentiation and inducing osteoblast apoptosis [29, 30]. TIMP3 was reported to be associated with RA and arthritis based on a search of the U. S. National Library of Medicine database (MEDLINE) (http://www.ncbi.nlm.nih.gov/IEB/Research/Ace mbly/av.cgi) [31]. A case-control study in a Chinese Han population linked TIMP3 polymorphism with severe knee OA [32]. In this study, TIMP3 was detected to be dysregulated in patients with OA. Hence, we hypothesized that TIMP3 was involved with OA.

Hsa-miR-17-92 polycistron encodes a cluster of seven miRNAs derived from the c-myc regulated c13orf 25 locus at chromosome 13q31.3, including miR-17-5p [33]. MiR-17-5p was reported to have a role as a tumor suppressor in breast cancer cells and as a key regulator of the G1/S phase cell cycle transition [7, 34]. MiR-17$5 p$ may act as an oncogene or a tumor suppressor in different cellular contexts [7]. Although no report linked hsa-miR-17-5p with OA, it was a down-regulated miRNA that covered most DEmRNAs in this study. In addition, TLR7, CTSS and TIMP3 were detected to be target genes of hsa-miR-17-5p. Given the results of our analysis, we hypothesized that hsa-miR-17-5p may participate in OA by regulating TLR7, CTSS and TIMP3. 

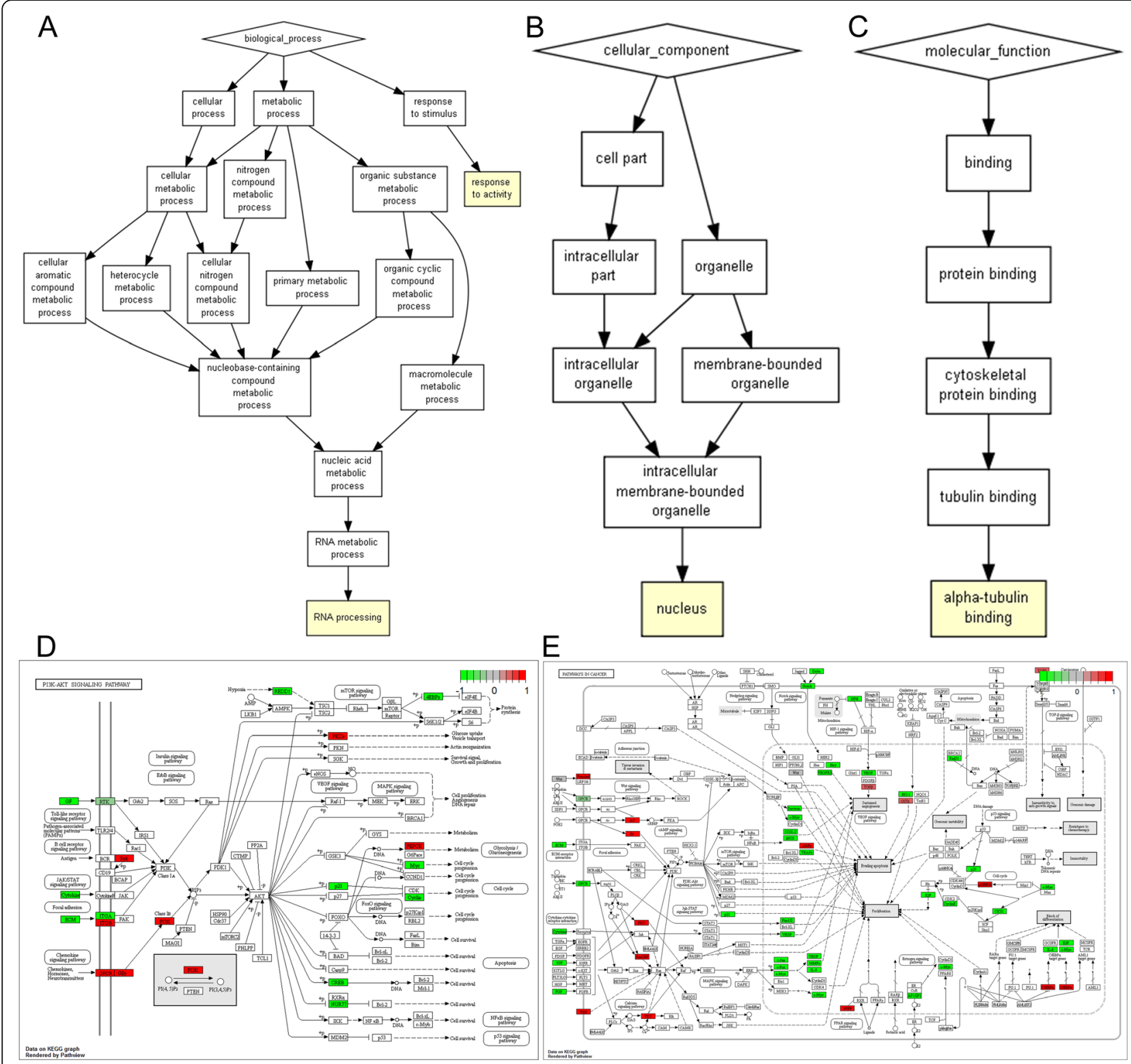

Fig. 5 Significantly enriched GO terms and KEGG pathways of DEmiRNA-target DEmRNAs. a. BP, biological process; b. CC, cellular component; $\mathbf{c}$. MF, molecular function. d-e KEGG pathways. d. PI3K-Akt signaling pathway. e. Pathways in cancer. The red and green rectangles represented the particles which regulated by the up- and down-regulated target DEmRNAs of DEmiRNAs, respectively. Appropriate copyright permission to use the signalling pathways was obtained

MiR-20b-5p is transcribed from the miR-106a 363 clusters which is reported to be involved in several process [35]. Ma et al. suggested that miR-20b-5p may play a vital role in multiple sclerosis (MS) pathogenesis [36]. In the study of Luo et al., miR-20b-5p was found to play role in promoting myoblast differentiation and repressing myoblast proliferation [35]. Interestingly, hsa-miR-20b-5p was down-regulated in patients with OA. In addition, TLR7 and CTSS were targeted by hsa-miR-20b-5p which indicated that
miR-20b-5p may implicate in OA. To our best knowledge, we are the first to report the role of hsa-miR20b-5p in OA.

\section{Conclusions}

In conclusion, we identified 1068 DEmRNAs (516 upregulated and 552 down-regulated DEmRNAs), 21 DEmiRNAs (6 up-regulated and 15 down-regulated DEmiRNAs) and 395 DEmiRNA-DEmRNA pairs in synovial tissues of patients with $\mathrm{OA}$, and emphasized 


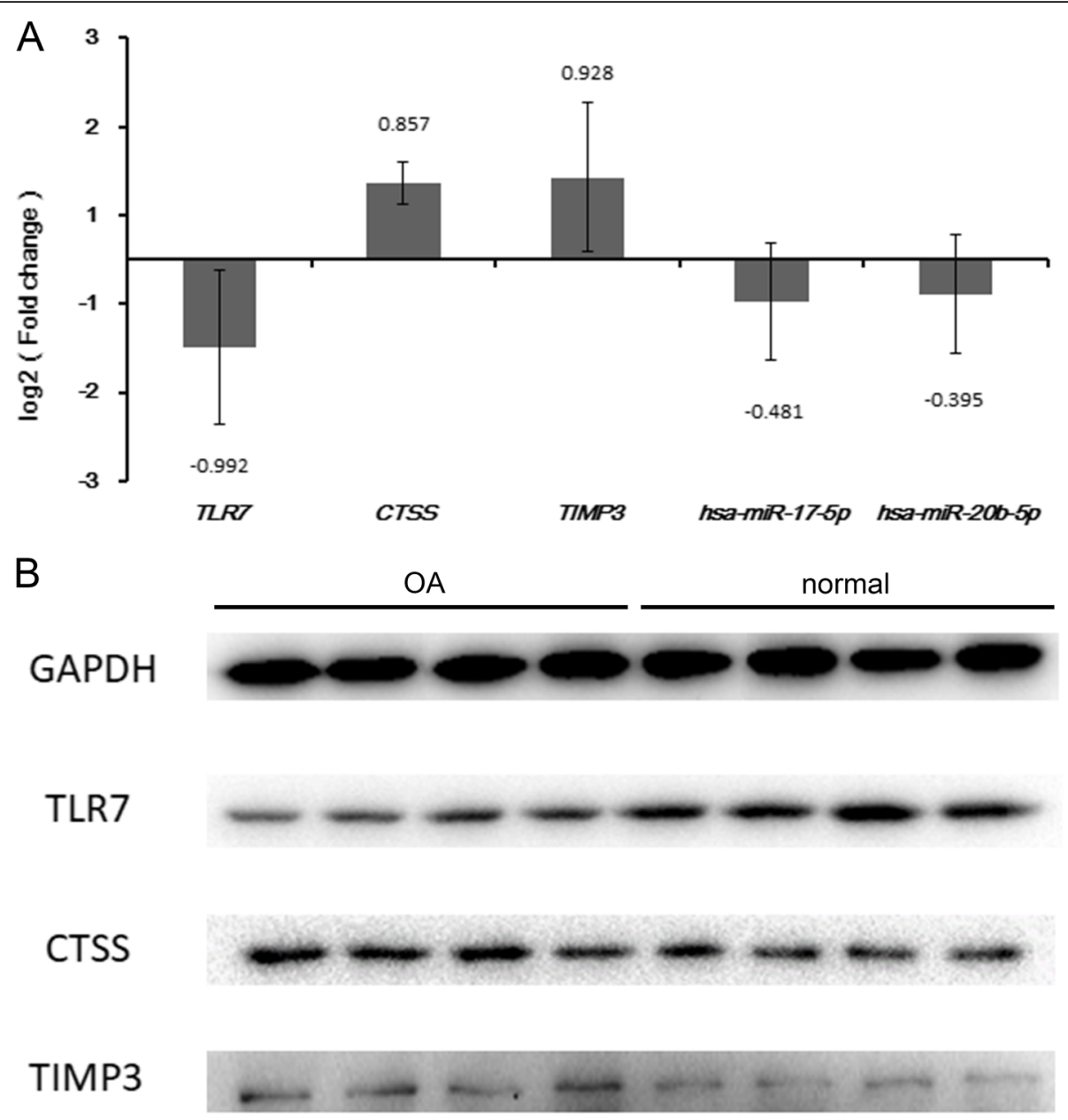

Fig. 6 The results of qRT-PCR and western blot. a. The quantitative real time polymerase chain reaction (qRT-PCR) results of the DEmRNAs and DEmiRNAs in OA. The $x$-axis represents the DEmRNAs/DEmiRNAs and the $y$-axis represents $\log _{2}$ (fold change). $\mathbf{b}$. Western blotting analysis of TLR7, CTSS and TIMP3 protein levels in OA and normal controls. GAPDH protein was used as the loading control

the importance of several mRNAs and miRNAs which may implicate in OA. These findings may provide new avenue to understand the mechanism of OA. A limitation of present study is the small sample size for RNA sequencing. The exact function of these mRNAs and miRNAs in OA need to be determined with further large sample research.

\section{Supplementary information}

Supplementary information accompanies this paper at https://doi.org/10. 1186/s12881-020-0978-5.

Additional file 1 : Figure S1 Flow chart of the analyses.

\section{Abbreviations}

CTSS: Cathepsin S; DEmiRNA: Differentially expressed miRNA; DEmRNA: Differentially expressed mRNA; ECM: Extracellular matrix; EDTA: Ethylenediaminetetraacetic acid; FPKM: Per million fragments mapped; GO: Gene Ontology; KEGG: Kyoto Encyclopedia of Genes and Genomes; OA: Osteoarthritis; PAMPs: Pathogen-associated molecular patterns; PCR: Polymerase chain reaction; PPI: Protein-protein interaction network;
RA: Rheumatoid arthritis; RIN: RNA integrity number; ssRNA: Single-stranded RNA; TIMP3: TIMP metallopeptidase inhibitor 3; TLR7: Toll like receptor 7

Acknowledgements

Not applicable,

\section{Authors' contributions}

$Y Z, Z W, X C, J Z, L Y$ and $S L$ analyzed and interpreted the data. $Y Z$ and $Z W$ were major contributors in writing the manuscript. $Y L$ designed the project. All authors read and approved the final manuscript.

\section{Funding}

This study was supported by Scientific Research Project of Sichuan Provincial Health and Family Planning Commission of China (17ZD023) and 2018 Science and Technology Plan Projects of Deyang City (2018SZS091). The funding body had no role in the design of the study and collection, analysis, and interpretation of data and in writing the manuscript.

\section{Availability of data and materials}

The raw-data have been uploaded to Gene Expression Omnibus (GEO)

(GSE143514, https://www.ncbi.nlm.nih.gov/geo/query/acc.cgi?acc=GSE143514). 


\section{Ethics approval and consent to participate}

This study was approved by the ethical committee of People's Hospital of Deyang City. Written informed consent for use of their samples from every participant were provided in the present study.

\section{Consent for publication}

Not applicable.

\section{Competing interests}

The authors declare that they have no competing interests.

Received: 31 May 2019 Accepted: 19 February 2020

Published online: 02 March 2020

\section{References}

1. Pereira D, Ramos E, Branco J. Osteoarthritis. Acta Medica Port. 2015;28(1):99106.

2. Mendel OI, Luchihina LV, Mendel W. Aging and osteoarthritis. Chronic nonspecific inflammation as a link between aging and osteoarthritis (a review). Adv Gerontol. 2015;5(4):252-60.

3. Fernández-Torres J, Zamudio-Cuevas Y, López-Reyes A, Garrido-Rodríguez D, Martínez-Flores K, Lozada CA, et al. Gene-gene interactions of the Wnt/ $\beta$ catenin signaling pathway in knee osteoarthritis. Molecular Biology Reports. 2018.

4. Goldring MB, Otero M. Inflammation in osteoarthritis. Curr Opin Rheumatol. 2011:23(5):471-8

5. Kolhe R, Hunter M, Liu S, Jadeja RN, Pundkar C, Mondal AK, et al. Genderspecific differential expression of exosomal miRNA in synovial fluid of patients with osteoarthritis. Sci Rep. 2017;7(1):2029.

6. Ren $Y M$, Zhao $X$, Yang T, Duan YH, Sun YB, Zhao WJ, et al. Exploring the key genes and pathways of osteoarthritis in knee cartilage in a rat model using gene expression profiling. Yonsei Med J. 2018:59(6):760

7. Cloonan N, Brown MK, Steptoe AL, Wani S, Chan LW, Forrest AR, et al. The miR-17-5p microRNA is a key regulator of the G1/S phase cell cycle transition. Genome Biol. 2008;9(8):1-14.

8. Lee RC, Feinbaum RL, Ambros V. The C. elegans heterochronic gene lin-4 encodes small RNAs with antisense complementarity to lin-14. Cell. 1993; 75(5):843.

9. Nugent M. MicroRNAs: exploring new horizons in osteoarthritis. Osteoarthr Cartil. 2016;24(4):573-80.

10. Shigeru M, Hiroshi A. Macro view of microRNA function in osteoarthritis. Nat Rev Rheumatol. 2012;8(9):543.

11. Wang Y, Wu N, Liu J, Wu Z, Dong D. FusionCancer: a database of cancer fusion genes derived from RNA-seq data. Diagn Pathol. 2015;10:131.

12. Karlsson C, Dehne T, Lindahl A, Brittberg M, Pruss A, Sittinger M, et al. Genome-wide expression profiling reveals new candidate genes associated with osteoarthritis. Osteoarthr Cartil. 2010;18(4):581-92.

13. Murata K, Yoshitomi H, Tanida S, Ishikawa M, Nishitani K, Ito H, et al. Plasma and synovial fluid microRNAs as potential biomarkers of rheumatoid arthritis and osteoarthritis. Arthritis Res Ther. 2010;12(3):R86 12,3(2010-05-14).

14. Christian B, Anna Z, Neng-Yu L, Arnd K, Carlo P, Annamaria I, et al. Signature of circulating microRNAs in osteoarthritis. Ann Rheum Dis. 2015;74(3):e18.

15. Altman $R_{1}$., Asch $E_{1}$, Bloch $D_{1}$. , Bole $G_{1}$., Borenstein $D_{1}$., Brandt $K_{1 .,}$ et al. Development of criteria for the classification and reporting of osteoarthritis. Classification of osteoarthritis of the knee. Diagnostic and therapeutic criteria Committee of the American Rheumatism Association. Arthritis Rheum 1986;29(8):1039-1049.

16. Kanehisa M, Sato Y, Furumichi M, Morishima K, Tanabe M. New approach for understanding genome variations in KEGG. Nucleic Acids Res. 2019;47(D1): D590-d5.

17. Goldring MB, Goldring SR. Articular cartilage and subchondral bone in the pathogenesis of osteoarthritis. Ann N Y Acad Sci. 2010;1192:230-7.

18. Delneste $Y$, Beauvillain $C$, Jeannin $P$. Innate immunity: structure and function of TLRs. Med Sci. 2007;23(1):67-73.

19. Gómez R, Villalvilla A, Largo R, Gualillo O, Herrero-Beaumont G. TLR4 signalling in osteoarthritis-finding targets for candidate DMOADs. Nat Rev Rheumatol. 2015;1 1(3):159-70.

20. Beutler B. Inferences, questions and possibilities in toll-like receptor signalling. Nature. 2004;430(6996):257-63.

21. Courtney W, Michael G. Recognition of viruses by cytoplasmic sensors. Curr Opin Immunol. 2010;22(1):41-7.
22. Scanzello CR, Goldring SR. The role of synovitis in osteoarthritis pathogenesis. Bone. 2012;51(2):249-57.

23. Chamberlain ND, Seung-Jae K, Vila OM, Volin MV, Suncica V, Pope RM, et al. Ligation of TLR7 by rheumatoid arthritis synovial fluid single strand RNA induces transcription of TNFa in monocytes. Ann Rheum Dis. 2013;72(3): 418-26.

24. Mar C, Yasmina J, Selene PG, Rebeca J, Pablos JL, Gomariz RP, et al. RNA sensors in human osteoarthritis and rheumatoid arthritis synovial fibroblasts: immune regulation by vasoactive intestinal peptide. Arthritis Rheum. 2014; 63(6):1626-36.

25. Hsing LC, Rudensky AY. The lysosomal cysteine proteases in MHC class II antigen presentation. Immunol Rev. 2005;207:229-41.

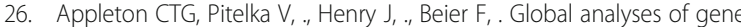
expression in early experimental osteoarthritis. Arthritis Rheum 2010;56(6): 1854-1868.

27. Lambert C, Dubuc JE, Montell E, Vergés J, Munaut C, Noël A, et al. Gene expression pattern of synovial cells from inflammatory and normal areas of osteoarthritis synovial membrane. Arthritis Rheum. 2014;66(4):960-8.

28. Troeberg L, Lazenbatt C, Anower EKMF, Freeman C, Federov O, Habuchi $H_{t}$ et al. Sulfated glycosaminoglycans control the extracellular trafficking and the activity of the metalloprotease inhibitor TIMP-3. Chem Biol. 2014;21(10): $1300-9$.

29. Han B, Wang HC, Li H, Tao YQ, Liang CZ, Li FC, et al. Nucleus pulposus mesenchymal stem cells in acidic conditions mimicking degenerative intervertebral discs give better performance than adipose tissue-derived mesenchymal stem cells. Cells Tissues Organs. 2014;199(5-6):342.

30. Yuan LQ, Liu YS, Luo XH, Guo L, Xie H, Lu Y, et al. Recombinant tissue metalloproteinase inhibitor-3 protein induces apoptosis of murine osteoblast MC3T3-E1. Amino Acids. 2008;35(1):123-7.

31. Tong Z, Liu Y, Chen B, Yan L, Hao D. Association between MMP3 and TIMP3 polymorphisms and risk of osteoarthritis. Oncotarget. 2017;8(48):83563-9.

32. Su SL, Yang HY, Lee HS, Huang GS, Lee CH, Liu WS, et al. Gene-gene interactions between TGF-1 $\left.\right|^{2} / S m a d 3$ signalling pathway polymorphisms affect susceptibility to knee osteoarthritis. BMJ Open. 2015;5(6):e007931.

33. Lin H. J Michael T, Michael T H, Eva H-M, David M, summer G, et al. A microRNA polycistron as a potential human oncogene. Nature. 2005; 435(7043):828-33.

34. Anwar H, Macus TK, Grady FS. Mir-17-5p regulates breast cancer cell proliferation by inhibiting translation of AIB1 mRNA. Mol Cell Biol. 2006: 26(21):8191-201.

35. Wen L, Li G, Yi Z, Nie Q, Zhang X. E2F1-miR-20a-5p/20b-5p auto-regulatory feedback loop involved in myoblast proliferation and differentiation. Sci Rep. 2016;6:27904

36. Xinting $M$, Juhua Z, Yin Z, Linlin J, Ping $M$, Yanmin L, et al. Expression, regulation and function of microRNAs in multiple sclerosis. Int J Med Sci. 2014;11(8):810.

\section{Publisher's Note}

Springer Nature remains neutral with regard to jurisdictional claims in published maps and institutional affiliations.

Ready to submit your research? Choose BMC and benefit from:

- fast, convenient online submission

- thorough peer review by experienced researchers in your field

- rapid publication on acceptance

- support for research data, including large and complex data types

- gold Open Access which fosters wider collaboration and increased citations

- maximum visibility for your research: over $100 \mathrm{M}$ website views per year

At $\mathrm{BMC}$, research is always in progress.

Learn more biomedcentral.com/submissions 